Voix et Images

voixetimages

\title{
Les Poètes de la Confédération dans les Confitures de coings de Jacques Ferron
}

\section{Guy Monette}

Volume 8, numéro 3, printemps 1983

Jacques Ferron

URI : https://id.erudit.org/iderudit/200398ar

DOI : https://doi.org/10.7202/200398ar

Aller au sommaire du numéro

Éditeur(s)

Université du Québec à Montréal

ISSN

0318-9201 (imprimé)

1705-933X (numérique)

Découvrir la revue

Citer cet article

Monette, G. (1983). Les Poètes de la Confédération dans les Confitures de coings de Jacques Ferron. Voix et Images, 8(3), 421-426.

https://doi.org/10.7202/200398ar d'utilisation que vous pouvez consulter en ligne.

https://apropos.erudit.org/fr/usagers/politique-dutilisation/ 


\title{
Les Poètes de la Confédération dans les Confitures de coings de Jacques Ferron
}

\author{
par Guy Monette, College royal militaire de Kingston
}

aे A.M. Hayward, I'Autre

L'identification des personnes réelles dont un auteur a pu se servir dans l'élaboration d'un personnage romanesque (les "clefs"l ou les remarques d'ordre anecdotique au sujet d'un écrivain et de son oeuvre ne remplaceront jamais une critique des textes et ne sauraient donner une information essentielle sur le caractère d'une oeuvre d'art spécifique; toutefois, mème dans leurs horizons limités, elles contribuent à enrichir un magma culturel propice à la vie littéraire.

Ainsi, personne ne s'étonnera qu'on reconnaisse Frank $R$. Scott, ancien doyen de la faculté de droit de l'Université McGill, dans le personnage de Frank Archibald Campbell des Confitures de coings et de la Charrette puisque Jacques Ferron lui-même admet cette "clef"1 qui vaut également pour le Frank-Anacharcis Scot du Ciel de Québec. L'évidence de cette clef cache cependant beaucoup plus qu'une simple facétie de la part de Jacques Ferron. Ce Frank (autant le réel que l'imaginaire) devient, aux yeux de Ferron, un "membre de "la» minorité dominante"2 au Québec, un "Rhodé sien bien intentionné, plus pernicieux qu'un autre" ${ }^{\prime 3}$. En ce sens, il joue, dans une oeuvre qui prêche la libération socio-politique des Québécois, le rôle d'un obstacle et d'une contrainte à éliminer. Voilà donc une "clef" qui ouvre une porte idéologiquement significative; toutefois, cette "clef" donne aussi accès à un phénomène fascinant d'intertextualité et son panneton comporte une dent spécifiquement littéraire.

En effet, les Confitures de coings, texte de langue française, s'ouvrait, dans sa première version intitulée la Nuit, sur une épigraphe en langue anglaise attribuée à Duncan Campbell Scott:

\section{What a night!}

Something was almost ritual about it! DUNCAN CAMPBELL SCOTH (sic) ${ }^{4}$

Certe version de l'oeuvre se terminait par l'évocation, en français évidemment, d'un "beau jour":

Comme toute conclusion est sommaire, je finis par me contenter d'une formule: à grande nuit, beau jour. J'en remettais la suite aux années qui me restaient à vivie ${ }^{5}$. 
Ainsi, à la nuit anglaise initiale succèderait le jour français final qui serait préférable à cette nuit, fût-elle "rituelle": "Je me retournai; Marguerite m'envoyait la main, une main de cinq doigts, joyeuse, la main du jour et non plus celle de la nuit au geste rituel"'6.

Or, il est remarquable que cette épigraphe et la nuit rituelle anglaise qu'elle évoque, aient été éliminées dans les Confitures de coings puisque, en transformant la Nuit en les Confitures de coings, la deuxième version affirme vouloir insister sur le poison que le narrateur aurait fait ingurgiter à Frank et sur la nécessité d'éliminer le pouvoir des "Rhodésiens" " Cette deuxième version du roman "se situe à la suite de Two Solitudes'B:

...à un moment de l'histoire où ces deux solitudes, tentative d'évitement qui a duré deux siècles, semblable à celle de deux bandes de singes hurleurs parvenant à partager un même territoire de chasse, prévenus par leurs cris, en ne se rencontrant jamais, deviennent incongrues par l'extension naturelle du français, rejoignant après 1960 toutes ses possibilités... 9

II apparaît alors logique que "la nuit réelle" de l'épigraphe de Duncan Campbell Scott, et cette épigraphe même, ne trouvent plus place dans les Confitures de coings, version qui accentue la nécessité de se débarrasser du pouvoir de la minorité dominante et de ses représentants.

Est-ce a dire que la lutte de libération ou, pour parler plus justement, l'urgence de repousser la présence d'un Autre sur un territoire qu'on réclame comme sien, s'étendrait même à l'imaginaire de cet Autre dans la mesure où il empiète sur l'imaginaire québécois? Si l'on se fie à l'étonnant jeu de citations que contiennent la Nuit et les Confitures de coings, il semble qu'on doive répondre affirmativement à cette question.

Le texte de Duncan Campbell Scott mis en exergue au roman paraitrait, de prime abord, s'inscrire dans la logique de la "clef" et entrainerait à conclure qu'il s'agit d'un extrait des oeuvres du père de Frank R. Scott puisque le Frank du roman, à qui il sert de modèle, cite constamment des poèmes de son père: "Il les savait donc par coeur, les poèmes de son vénérable père!"10. Toutefois, un problème se pose immédiatement: le père de Frank R. Scott n'est pas Duncan Campbell Scott mais bien Frederick George Scott, également poète. Simple question d'homonymie: l'un et l'autre poètes auraient été confondus comme étant le père de Frank Scott et l'on serait tout uniment renvoyé à la "clef".

Or, il n'en est rien. En effet, les Confitures de coings (1972 et 1977) et la Nuit (1965 et 1979) mettent dans la bouche de Frank des extraits de poèmes de Duncan Campbell Scott, de Bliss Carman, de Frederick George Scott et de Charles G.D. Roberts; et tous ces extraits sont attribués au père de Frank! p. 54):

Ainsi, les vers cités à la page 163 des Confitures de coings 11972; la Nuit (1965),

But his eyes were jewel (sic) of content

Set in circles of peace ${ }^{11}$

sont attribués au père de Frank mais sont dus à la plume de Duncan Campbell Scott et extraits du poème "On the Way to the Mission"12. 
Les quatre vers mentionnés à la page 164 des Confitures de coings (1972), dont les deux derniers sont repris presque textuellement à la page 166 Va Nuit (1965), pp. 54 et 56I, sont attribués au père de Frank:

These mountains reign alone, they do not share

The transitory life of woods and streams;

Wrapt in the deep solemnity of dreams,

They drain the sunshine of the upper air ${ }^{13}$.

IIs ont en fait été écrits par Frederick George Scott, père de Frank R. Scott, et proviennent du poème intitulé "The Laurentians"14.

Les vers que cite $F$.ank à la page 167 des Confitures de coings $(1972$; la Nuit (1965), p. 58) sont tirés de "Kinship", poème de Sir Charles G.D. Roberts, bien que le texte les attribue toujours au père de Frank ${ }^{15}$ :

Back to Knowledge and renewal

Faith to fashion and reveal

Take me mother, in compassion

All thy hurt ones fail to heal ${ }^{16}$

Quant aux vers que Frank récite à la page 185 des Confitures de coings (1972; la Nuit (1965), p. 79) et qui, selon le texte, sont de son père, ils sont l'oeuvre de Bliss Carman et tirés du poème "Low Tide on Grand Pré"17.

Was it a year or lives ago

We took the grasses in our hands

And caught the summer flying low... ${ }^{18}$

Les Confitures de coings se transforme alors en instrument de guérilla culturelle car ces citations constituent en fait un florilège des oeuvres des Poètes de la Confédération. Ces poètes, ainsi appelés parce qu'ils sont nés vers 1867, ont chanté en langue anglaise les paysages du Québec et du Canada dans des vers patriotiques.

Les oeuvres des Poètes de la Confédération ${ }^{19}$ se voient récusées et tournées en dérision parce que, comme les oeuvres du père de Frank, elles ne cerneraient pas la vie et constitueraient une appropriation abusive et illusoire du domaine québécois:

Son père le pasteur anglican ${ }^{20}$ qui, en toute sérénité, écrivait des vers au milieu de la tranquillité québécoise et des collines laurentiennes rehaussées pour les besoins du poème.

These mountains reign alone, they do not share

The transitory life of woods and streams;

Wrapt in the deep solemnity of dreams

They drain the sunshine of the upper air.

Un imbécile"2!

II écrivait des poèmes qui obéissaient à toutes les règles, des poèmes bien aussi forts que le filet du pécheur; il voulait lui aussi capter la vie qui lui échappait pour la fixer en anglais. La Vie qui coulait en français de l'autre côté du mur22. 
Toute la poésie attribuée au père de Frank, et qui en fait équivaut à l'univers littéraire des Canadiens anglais, se voit ainsi expulsée du territoire québécois parce qu'elle n'y est pas pertinente.

De plus, l'univers littéraire anglophone que dénonce les Confitures de coings aurait tendance à dévaloriser et à méconnaître les Québécois. En effet, après avoir cité en français, et en les identifiant clairement cette fois, quelques vers du poème satirique de Samuel Butler intitulé "A Psalm of Montreal"23 dans lequel Butler se gausse de l'étroitesse d'esprit et du puritanisme de Montréalais qui, selon le contexte, sont de langue anglaise, le narrateur des Confitures de coings fera un véritable procès d'intention aux anglophones:

- O Dieu! O Montréal! fis-je, ce n'est pas dans les habitudes de vos compatriotes de se déprécier: ils peuvent tout mettre à notre compte en fait de ridicules et de sottises. Il doit exister une version de ce poème où le beau-frère du mercier de Monsieur Spurgeon est un brave CF, victime de ses curés.

- Possible, dit Frank.

Cela ne l'avait pas empêché de me le réciter ${ }^{24}$.

Ce passage, qui n'apparaissait pas dans la Nuit, accentue encore davantage le durcissement des positions face à l'univers imaginaire de l'Autre dans les Confitures de coings 25 .

D'ailleurs, le père de Frank, selon les Confitures de coings, aurait contribué à faire changer le nom original de la ville de Rivière-du-loup en Louiseville pour honorer la princesse Louise, fille de la reine Victoria ${ }^{26}$. Voilà qui constitue un exemple d'envahissement culturel du domaine québécois par la minorité dominante anglaise, exemple d'autant plus significatif qu'il porte sur le nom de la ville natale du narrateur. Ainsi, les Confitures de coings attaque et récuse l'univers culturel et imaginaire des anglophones parce que ce dernier investit abusivement le domaine des francophones et le fausse. Plus qu'une simple "clef", la présence de Frank R. Scott derrière le personnage de Frank Archibald Campbell renvoie en fait, par l'entremise de Frederick George Scott, des Poètes de la Confédération (D.C. Scott, Bliss Carman, Charles G.D. Roberts) et de Samuel Butler, à tout l'imaginaire des anglophones. Ce véritable travail de sape vise la culture canadienne, non pas parce qu'elle serait en soi mauvaise et répréhensible mais parce qu'elle est déplacée au Québec et fausse la réalité historique dans laquelle le texte souhaite inscrire l'homme québécois.

Une "interpolation" du narrateur (qui se confond ici avec l'auteur) empêche le texte de glisser dans le racisme ou le chauvinisme. Les Confitures de coings reste en effet tenté par un humanisme anhistorique dans lequel l'art s'inscrirait sans doute:

Frank, que serons-nous aux yeux de cet homme nouveau, sur le point d'apparaitre? Verrat-il une différence entre un Écossais et un Canadien français? J'en doute, et si j'ai peine à te haîr, c'est peut-être que ma haine est déjà périmée... ${ }^{27}$

Toutefois, cette utopie n'empêche pas les Confitures de coings d'assumer le hic et nunc québécois et d'en explorer toutes les conséquences.

Cette utilisation habile de textes de poètes canadiens témoigne d'une culture fort diversifiée et étonnamment assimilée à la mode québéco-ferronienne ${ }^{28}$; mais elle 
affirme surtout, et ce au niveau même de l'univers littéraire, que seul le français peut exprimer l'imaginaire du pays québécois.

1. Voir "Appendice aux Confitures de coing (sic) ou le Congédiement de Frank Archibald Camp:bell" dans: Jacques Ferron, les Confitures de coing (sic) et autres textes, Montréal, Parti pris, coll. "Paroles", 1972, pp. 263-266. Les Confitures de coings et autres textes suivi de le Journal des Confitures de coings (Montréal, Parti pris, coll. "Projections libérantes", 1977) reproduit les textes de 1972 en y ajoutant des extraits de la critique.

2. Les Confitures de coings (1972), p. 263.

3. Ibid.

4. Cette épigraphe qui apparaissait dans la Nuit (Montréal, Parti pris, coll. "Paroles", 1965) a été éliminé dans les éditions de 1972 et 1977 de la deuxième version du roman dont le titre est devenu les Confitures de coings. Diane Potvin, qui reproduit l'édition originale de la Nuit dans la collection "Classiques du monde" Va Nuit de Jacques Ferron, présentée par Diane Potvin, Paris/Montréal, Éditions France Québec/Fernand Nathan, 1979), reprend cette épigraphè à la page 18, mais en traduction française: "'Quelle nuit! II y avait en elle quelque chose de quasi rituel." Duncan Campbell Scort. Nous $n$ 'avons pas réussi à retracer ce texte dans l'oeuvre de Duncan Campbell Scort.

5. Jacques Ferron, la Nuit (1965), p. 134. La Nuit (1979), p. 103. Ces deux phrases lla pénultième et l'antépénultième du roman) sont éliminées dans les éditions (1972 et 1977) des Confitures de coings.

6. La Nuit (1965), pp. 133-134. La Nuit (1979), p. 103. Cette phrase est conservée dans les éditions des Confitures de coings (1972 et 1977 ) mais elle y occupe l'avant-dernière position, ce qui la met plus en relief.

7. Voir les Confitures de coings (1972), pp. 265-266.

8. Roman de Hugh McLennan dàns lequel l'univers d'un Canadien français de vieille famille seigneuriale est détruit au contact des Anglo-saxons.

9. Les Confitures de coings (1972), pp. 263-264.

10. Les Confitures de coings (1972), p. 185. La Nuir (1965), p. 79.

11. Diane Potvin traduit par: "Mais ses yeux étaient des joyaux de sérénité/ Sertis dans un écrin de paix" (La Nuit, 1979, p. 48).

12. On retrouvera ce poeme dans Poets of the Confederation, edited by Malcolm Ross, Toronto, McClelland \& Stewart, coll. "New Canadian Library Original", 1960, p. 98.

13. Diane Potvin traduit par: "Ces monts règnent seuls, ils ne partagent point/ La vie passagère des bois et des vents;/ Drapés dans la solennité profonde des rêves, / lls filtrent du haut de I'air la lumière du soleil" (La Nuit, 1979, p. 49). Nous traduirions, quant à nous, "streams" par "ruisseaux".

14. On retrouvera ce poème dans Poems de Frederick George Scott, London, Constable, 1910, p. 257.

15. On retrouvera ce poème dans Selected Poems of Sir Charles G.D. Roberts, Toronto, Ryerson Press, 1936, p. 78. Le texte original porte "fain" au lieu de "fail" dans le dernier vers.

16. Diane Potvin traduit par: "Revenons à la sagesse et au renouveau,/ Croyons à la forme et à la révélation,/ Prends-moi en pitié, Mère,/ Toute ta blessure qu'on a oublié de panser" "Lo Nuit, 1979, p. 511. La traduction du dernier vers est fautive et devrait se lire "Daigne guérir tous tes enfants blessés" selon l'original ("fain"l et "Ne réussis pas à guérir tous les enfants blessés" selon la version avec "fail". Diane Potvin a sans doute voulu rester fidèle au texte tel que cité dans la Nuit et dans les Confitures de coings, mẻme s'il est grammaticalement très insolite, sinon incorrect, en anglais.

17. On retrouvera ce poème dans Poets of the Confederation, p. 26.

18. Diane Potvin traduit (en éliminant la construction interrogative) par: "Il y a un an ou très longtemps/ Nous avons pris les herbes dans nos mains/ Et nous avons saisi l'été qui volait 
bas..." (La Nuit, 1979, p. 66).

19. Au point de vue idéologique, il n'y a d'ailleurs qu'un pas vite franchi entre les pères-Poètes de la Conféderation et les Pères de la Confédération.

20. Frederick George Scott était pasteur anglican et devint chanoine ("Canon").

21. Les Confitures de coings (1972), p. 164. La Nuit (1965), p. 54.

22. Les Confitures de coings (1972), pp. 184-185. La Nuit (1965), p. 79.

23. On retrouvera l'original en anglais dans The Blasted Pine. An Anthology of Satire, Invective and Disrespectful Verse Chiefty by Canadian Writers, edited by F.R. Scott and A.J.M. Smith, Toronto, Macmillan, 1967. p. 62. Samuel Butter est un Ecrivain de la Grande-Bretagne.

24. Les Confitures de coings (1972), p. 184.

25. Ajoutons que la traduction d'un des vers du poème de Butler (traduction que le texte n'attribue plus a Frank) est devenue plus française: '"Qui prépares aussi les peaux des canadiennes chouettes" (La Nuit, 1965, p. 78) se transforme en "Empailleur attitré de toutes les chouettes canadiennes" (Les Confitures de coings, 1972, p. 183).

26. Voir les Confitures de coings (1972), o. 190.

27. Les Confitures de coings (1972), pp. 186-187. La Nuit (1965), p. 81. .

28. Le sort qui est fait aux oeuvres des Poètes de la Confédération reléve certes d'une volonté malicieusement parodique; mais encore fallait-il connaitre ces textes (peut-être même les apprécier) pour les assimiler aussi judicieusement et les intégrer à une oeuvre littéraire qué bécoise dans laquelle ils sont loin d'être un simple hors-d'oeuvre.

Nous remercions D. Spertigue de Queen's University qui, par personne interposee, nous a mis sur cette piste qu' avait à peine entrevue Ray Ellenwood, le traducteur des Confitures de coings: "Dans les Confitures de coings, par exemple, (Ferron) utilise des poèmes de Samuel Butler (identifié) et de Bliss Carman et D.C. Scott lattribués indistinctement au père Frank Archibald Campbell" IQuince Jam by Jacques Ferron, translated by Ray Ellenwood, Toronto, Coach House Quebec Translations, 1977, p. 9). C'est nous qui traduisons. 